\title{
Association of Cytokeratin and Vimentin Protein in the Genesis of Transitional Cell Carcinoma of Urinary Bladder Patients
}

\author{
Arshad H. Rahmani, ${ }^{1}$ Ali Y. Babiker, ${ }^{1,2}$ Wanian M. AlWanian, ${ }^{1}$ Shawgi A. Elsiddig, \\ Hassan E. Faragalla, ${ }^{5,6}$ and Salah M. Aly ${ }^{7}$ \\ ${ }^{1}$ Department of Medical Laboratories, College of Applied Medical Sciences, Qassim University, Qassim 51452, Saudi Arabia \\ ${ }^{2}$ Department of Histopathology and Cytology, College of Medical Laboratories Science, University of Sciences and Technology, \\ P.O. Box 407, Omdurman, Sudan \\ ${ }^{3}$ Faculty of Applied Medical Sciences, Aljouf University, P.O. Box 2014, Sakaka, Saudi Arabia \\ ${ }^{4}$ Faculty of Medical Laboratory Sciences, Omdurman Islamic University, 14415 Omdurman, Sudan \\ ${ }^{5}$ College of Medical Laboratories Science, Sudan University of Sciences and Technology, Khartoum, Sudan \\ ${ }^{6}$ Department of Public Health, Health Sciences College, Umm Al-Qura University, Al Leith, Makkah 21421, Saudi Arabia \\ ${ }^{7}$ Faculty of Veterinary Medicine, Suez Canal University, Ismailia, Egypt
}

Correspondence should be addressed to Ali Y. Babiker; alibabkr99@gmail.com

Received 15 August 2015; Revised 26 October 2015; Accepted 28 October 2015

Academic Editor: Ja Hyeon Ku

Copyright (C 2015 Arshad H. Rahmani et al. This is an open access article distributed under the Creative Commons Attribution License, which permits unrestricted use, distribution, and reproduction in any medium, provided the original work is properly cited.

\begin{abstract}
The aim of study was to examine the localization and distribution of cytokeratin (CK) and vimentin protein and their association with clinical outcome of the TCC patients. Expression pattern of cytokeratin and vimentin was evaluated by immunohistochemistry in TCC cases and inflammatory lesions. Cytoplasmic expression of CK was noticed in $52.17 \%$ of TCC cases and its expression was not observed in inflammatory lesions of bladder specimens. Vimentin showed expression in $69.00 \%$ cases of TCC. Significant differences were noticed in expression pattern of CK and vimentin in inflammatory lesion and Transitional Cell Carcinoma cases. Vimentin expression increased with the grade of TCC and this difference was statistically significant whereas expression of CK decreased according to the grade of TCC. Furthermore, it was also observed that expression pattern of vimentin was high in $\geq 55$ years as compared to $<55$ age group patients and these differences were significant in men as compared to women. Expression pattern of CK did not show any significant relation with age and gender. Therefore, it can be concluded that cytokeratin and vimentin will be helpful markers in the early diagnosis of Transitional Cell Carcinoma/bladder carcinoma.
\end{abstract}

\section{Introduction}

Bladder carcinoma is one of the most common malignancies worldwide in term of morbidity and mortality. Despite of its high prevalence, the molecular mechanism involved in the induction of bladder carcinoma and its progression is not properly understood [1]. Altered expressions of various genes/protein such as tumour suppressor gene, oncogene, and apoptotic genes have been observed in several types of tumour [1, 2]. A range of tumour markers and therapy targets are in use to investigate the Transitional Cell Carcinoma (TCC) and its clinical outcome. But still potential marker is needed to diagnose/investigate the Transitional Cell
Carcinoma behaviour. Therefore, the assessment of potential biomarkers will be important move towards diagnosis and treatment of Transitional Cell Carcinoma. However, in this vista, intermediate filament family proteins play an important role in the genesis of Transitional Cell Carcinoma.

Cytokeratins are one of the chief structural proteins, which form the cytoplasmic network of intermediate filaments [3] and its family contains at least 20 types of cytoplasmic intermediate filaments found in epithelial cells [4]. They are expressed in a tissue-specific manner in normal organs as well as in the tumors that derived from them [5]. Different types of expression patterns of cytokeratins were noticed in carcinoma and normal/inflammatory lesions of bladder. 
A study report confirmed that $92 \%$ of benign/reactive cases were either CK20 (-) or $(+)$ only in the upper 1/3 urothelium whereas in dysplastic cases CK20 staining distribution was noticed as $60 \%$ in $2 / 3$ of the urothelium, $30 \%$ full thickness, $10 \%$ in the upper $1 / 3$ urothelium and among carcinoma in situ (CIS) cases, $89 \%$ had full thickness of CK20 positivity [6]. Another valuable study reported that all cases (100\%) of normal urothelia had normal expression patterns with Cytokeratin 20 and ninety-six percent of morphologically unequivocal cases of reactive urothelial atypia (RUA) showed normal expression patterns of Cytokeratin 20 whereas, in the carcinoma in situ (CIS) group, $86 \%$ had abnormal CK20 expression [7]. Previous finding confirmed that CK20 showed patchy cytoplasmic immunoreactivity in the superficial umbrella cell layer of the normal urothelium [8] and nonneoplastic urothelium showed no reactivity to CK20 except for umbrella cells [9].

Intermediate filaments are one of the three major cytoskeleton networks and these filaments consist of a number of different members such as vimentin and the cytokeratin proteins [10]. Vimentin shows important roles in cell adhesion, migration, and signalling [11]. Numerous studies described the vimentin reactive cells in benign and malignant breast tissues $[12,13]$ and vimentin expression in the tumour stroma was valuable in identifying colorectal cancer patients with a poor prognosis [14]. Different expression pattern of vimentin was also noticed in bladder cancer and normal urothelia. An important study reported that expression of vimentin was observed in $43 \%$ of bladder cancers, whereas it was not expressed or found negative in all normal urothelia [15]. The aim of study was to assess the expression profile of cytokeratin and vimentin in Transitional Cell Carcinoma and its association with clinical outcome such as sex, age, and grade of the tumour via immunohistochemistry.

\section{Materials and Methods}

2.1. Tissue Specimens. Forty-six patients with Transitional Cell Carcinoma (TCC) and ten cases of inflammatory lesions of bladder, confirmed by histopathologist, were taken to examine the expression profile of both markers and its interpretation with clinical outcome. The patient's details about age and sex were noted as range of $24-78$ years with mean age $36 \pm 12$ years and 38 male and 8 female cases. The TCC cases were further categorized as Grade I $(n=14)$, Grade II $(n=18)$, and Grade III $(n=14)$. Haematoxylin and eosin ( $\mathrm{H}$ and $\mathrm{E})$ staining was performed on each case to confirm the grading of the tumour.

\subsection{Immunohistochemical Detection of Cytokeratin and} Vimentin Protein. Transitional Cell Carcinoma (TCC) was sectioned with microtome into $5 \mu \mathrm{m}$ thick. Deparaffinized was performed with three changes of xylene and blocking of endogenous peroxidase activity was done via $0.3 \%$ hydrogen peroxide in methanol for 30 minutes. After the quenching step, antigen retrieval was made with citrate buffer $(\mathrm{pH}$ 6.0) in pressure cooker for 30 minutes and then sections were kept at room temperature for 20-30 minutes. Then, blocking step was made with blocking agent for 10 minutes

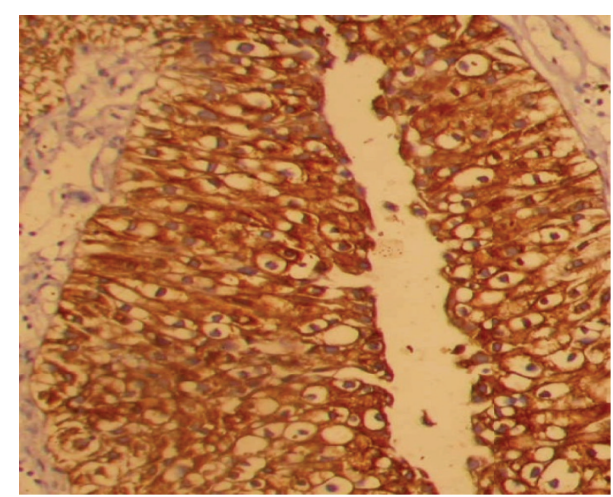

Figure 1: Cytoplasmic expression of cytokeratin protein in Transitional Cell Carcinoma (orig. mag $\times 100$ ).

and sections were washed with PBS. Monoclonal mouse antihuman cytokeratin (Clone AE1/AE3, Dako) and Monoclonal mouse Anti-Vimentin (Clone V9, Dako) antibodies was applied at 1:100 and 1:75 dilutions for 2 hours at room temperature in humid chamber. Following incubation with secondary antibody for 45 minutes, followed by incubation with streptavidin-biotin enzyme complex was applied for 30 minutes. Finally, diaminobenzidine (DAB) chromogen was used and then sections were counterstained with haematoxylin.

2.3. Evaluation of Staining. Negative (omission of antibody) and positive controls (oral cancer) cases were run to verify the quality of staining and confirmation of the procedures. Markers such as cytokeratin and vimentin were considered as positive if more than $5 \%$ of cells were positive and less than $5 \%$ cytoplasmic positivity was taken as negative. Cytoplasmic expression of cytokeratin and vimentin was considered as positive cases. All fields of the section were analyzed by two pathologists and more than 400 tumor cells, in five different area, were counted and mean percentage was calculated.

2.4. Statistical Analysis. Markers' expression and its association with clinical outcome were analyzed by Chi square $(\lambda)^{2}$. The $P$ value $P<0.05$ was considered as statistically significant.

\section{Results}

Cytokeratin and vimentin markers were analyzed according to age, sex, and histological grade and results were interpreted accordingly.

3.1. Immunohistochemical Analysis of Cytokeratin Protein. Cytokeratin expression was noticed in $24.00(52.17 \%)$ of TCC cases in cytoplasm (Figure 1) including 9 (64.28\%) in Grade I, 10 (55.55\%) in Grade II, and 5 (35.71\%) in Grade III whereas $22.00(47.82 \%)$ of TCC did not show any expressions of cytokeratin protein (Figure 2). Furthermore, the expression profile was examined according to age and sex and difference of expression pattern was insignificant 
TABLE 1: Expression of cytokeratin (CK) and vimentin in TCC and inflammatory lesion cases and its association with grade of the tumour.

\begin{tabular}{|c|c|c|c|c|c|c|}
\hline \multirow{2}{*}{ Clinical parameters patients } & \multicolumn{4}{|c|}{ CK expression } & \multicolumn{2}{|c|}{ Vimentin expression } \\
\hline & Total cases & $\%$ positivity & $P$ value & Positive cases & $\%$ positivity & $P$ value \\
\hline Inflammatory lesions & 10 & 00 & & 01 & 10.00 & \\
\hline \multicolumn{7}{|l|}{ Tumor grades } \\
\hline I & 9 & 64.28 & \multirow{4}{*}{$<0.05$} & 6 & 42.85 & \multirow{4}{*}{$<0.05$} \\
\hline II & 10 & 55.55 & & 13 & 72.22 & \\
\hline III & 5 & 35.71 & & 13 & 92.85 & \\
\hline Total cases & 24 & 52.17 & & 32 & 69.56 & \\
\hline \multicolumn{7}{|l|}{ Sex } \\
\hline Male & 20 & 52.60 & \multirow{2}{*}{$>0.05$} & 31 & 81.00 & \multirow{2}{*}{$<0.05$} \\
\hline Female & 04 & 50.00 & & 01 & 12.5 & \\
\hline \multicolumn{7}{|l|}{ Age } \\
\hline$<55$ years & 8 & 50.00 & \multirow{2}{*}{$>0.05$} & 7 & 43.00 & \\
\hline$\geq 55$ years & 16 & 55.00 & & 25 & 63.3 & \\
\hline
\end{tabular}

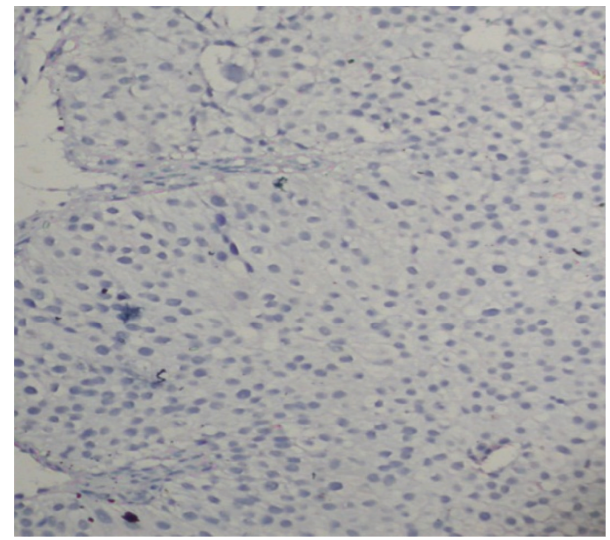

Figure 2: Cytokeratin did not show expression in Transitional Cell Carcinoma (orig. mag $\times 100)$.

(Table 1). Markedly, the positivity of CK decreased according to grade of the tumour, but this difference was statistically significant. Among inflammatory lesions of bladder cases, CK did not exhibit any expression. The expression pattern of CK was measured in both sexes and less than 50 years and equal to or more than 50 years age group, but differences in expression pattern of CK did not reach statistically significant level in gender and different age groups.

3.2. Positivity of Vimentin in TCC and Inflammatory Lesions of Bladder Cases. Vimentin expressions were analyzed in TCC cases and it was noticed that vimentin was overexpressed in $32(69.56 \%)$ of TCC cases (Table 1 and Figure 3 ) and 14 cases $(30.43 \%)$ did not show any expression of vimentin (Figure 4 ). One case out of 10 showed expression in inflammatory lesions of bladder. This difference of expression pattern in TCC and inflammatory lesions was statistically significant. Expression of vimentin was further categorized according to grade, gender, and sex. The positivity of vimentin increased according to grade of the TCC and it was (6 cases, $42.85 \%$ ), (13 cases, $72.22 \%$ ), and (13 cases, $92.85 \%$ ) in Grade I, Grade II and Grade III, respectively (Table 1). The expression pattern

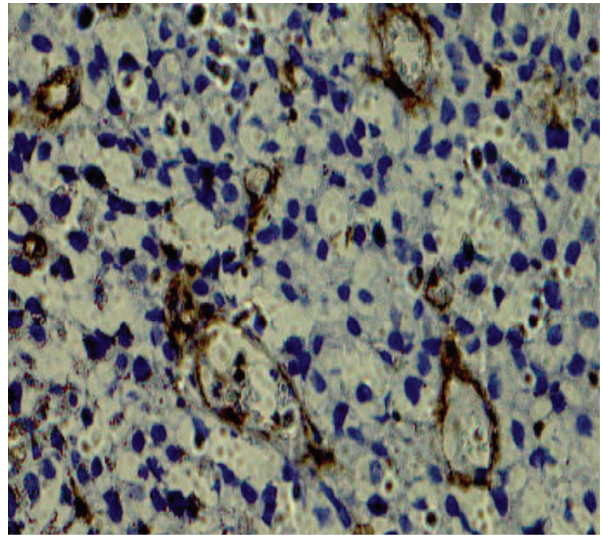

Figure 3: Vimentin showed expression in Transitional Cell Carcinoma (orig. mag $\times 100$ ).

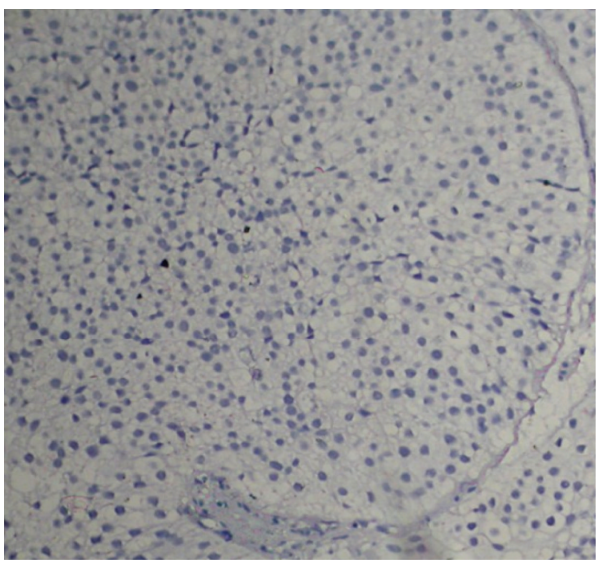

FIgURE 4: Vimentin did not show expression in Transitional Cell Carcinoma (orig. mag $\times 100$ ).

of vimentin was statistically significant according to grade. The TCC cases were divided into two groups on the basis of age, that is, $<55$ years and $\geq 55$ years, and it was observed that expression of vimentin was high in $\geq 55$ years as compared 
to $<55$ years and these differences were significant in men as compared to women (Table 1).

3.3. Correlation of Both Markers and Their Interpretation according to Age and Gender. Transitional Cell Carcinoma (TCC) cases were examined for both markers; most of the cases showed both $\mathrm{CK}$ and vimentin positivity and our results showed that cytokeratin and vimentin have pivotal role in development and progression of TCC. A negative correlation was observed in $\mathrm{CK}$ and vimentin expression with gradewise observations. Markedly, the positivity of CK decreased according to grade of TCC whereas vimentin increases according to the grade of the carcinoma.

\section{Discussion}

Bladder cancer is the fifth most common cancer worldwide and also one of the major causes of cancer morbidity and mortality [16]. It affects men more as compared to women $(3: 4.1)$ [17]. Our finding also showed similar pattern and most of TCC patients were male (38 cases, $82.60 \%)$ and ( 8 cases, $17.39 \%$ ) were female. The exact reason behind this is not understood, but it is thought that males acquire smoking, chewing, and drugs habits earlier than women. Currently, various markers are in practice to diagnose the Transitional Cell Carcinoma via immunohistochemistry. But still a potential marker is needed to diagnosis the early bladder carcinoma/Transitional Cell Carcinoma. In this vista, our study tried to find some more information of CK and vimentin role in the diagnosis of bladder carcinoma/Transitional Cell Carcinoma.

In our finding expression of cytokeratin was noticed in 24 cases of TCC (52.17\%) and CK was not expressed in inflammatory lesion of bladder cases. Furthermore, our results showed that the expression pattern of $\mathrm{CK}$ decreased according to the grade of the TCC (from Grades I to III) and these differences were statistically significant. Several findings showed different types of results in this issue and some results were in accordance with our study. One of the study results revealed that positivity of Cytokeratin 20 associated with increasing tumor grade and stage and it was observed that $69.4 \%$ cases of high grade tumors showed Cytokeratin 20 positivity as compared to $45.00 \%$ of other grades of tumours [18]. Another study reported that CK-20 expression was observed in all grades of tumour as $75.00 \%$ of low malignancy potential, $83.00 \%$ of low grade, $38.00 \%$ of high grade, and $67.00 \%$ of high grade tumors that invaded adjacent structures [19]. Other important study results showed that reduction or loss of cytokeratin expression was significantly correlated with tumor stage and grade [20]. Our finding did not reach significant level in terms of positivity of CK in gender and age basis analysis.

In the current study vimentin expressions were only noticed in cytoplasm and 32 cases (69.56\%) of TCC showed expression of vimentin. Previous finding also showed high expression in Transitional Cell Carcinoma cases. A study finding showed that $30(24.8 \%)$ were positive for vimentin in bladder cancer patients [21] and other important investigations showed that $43.00 \%$ bladder cancer expressed vimentin [15].

The present study results confirmed that vimentin expression is associated with the grade of the tumour. A recent study showed that expression of HMGA2, loss of E-cadherin, and expression of vimentin are significantly correlated with bladder cancer grade and stage [15]. Another study also confirms that vimentin was found to have statistically significant correlations with grade, recurrence, and progression [21]. Our results show that expression was different in different age group and it was noticed that older age group $(\geq 55)$ showed high expression $(63.00 \%)$ of vimentin especially in male. The exact reason is difficulty to explain the difference in expression pattern of both markers in cancer and inflammatory lesions. But it is thought that long cumulative exposure of carcinogen plays a critical role in the DNA damage/DNA adduct formation and alterations in transitional epithelial. The current study demonstrates that significant difference of expression pattern of both markers in TCC and inflammatory lesions of bladder and expression of vimentin was closely associated with the grade of TCC. Keeping in view the above information, our study concluded that use of both markers will be helpful in the diagnosis/investigation of early Transitional Cell Carcinoma.

\section{Conflict of Interests}

The authors declare that there is no conflict of interests.

\section{References}

[1] A. H. Rahmani, M. Alzohairy, A. Y. Y. Babiker, A. A. Khan, S. M. Aly, and M. A. Rizvi, "Implication of androgen receptor in urinary bladder cancer: a critical mini review," International Journal of Molecular Epidemiology and Genetics, vol. 4, no. 3, pp. 150-155, 2013.

[2] M. M. A. Rizvi, M. S. Alam, A. Ali, S. J. Mehdi, S. Batra, and A. K. Mandal, "Aberrant promoter methylation and inactivation of PTEN gene in cervical carcinoma from Indian population," Journal of Cancer Research and Clinical Oncology, vol. 137, no. 8, pp. 1255-1262, 2011.

[3] E. Fuchs and K. Weber, "Intermediate filaments: structure, dynamics, function, and disease," Annual Review of Biochemistry, vol. 63, pp. 345-382, 1994.

[4] J. Southgate, P. Harnden, and L. K. Trejdosiewicz, "Cytokeratin expression patterns in normal and malignant urothelium: a review of the biological and diagnostic implications," Histology and Histopathology, vol. 14, no. 2, pp. 657-664, 1999.

[5] S. Kummar, M. Fogarasi, A. Canova, A. Mota, and T. Ciesielski, "Cytokeratin 7 and 20 staining for the diagnosis of lung and colorectal adenocarcinoma," British Journal of Cancer, vol. 86, no. 12, pp. 1884-1887, 2002.

[6] I. Z. Yildiz, R. Recavarren, H. B. Armah, S. Bastacky, R. Dhir, and A. V. Parwani, "Utility of a dual immunostain cocktail comprising of p53 and CK20 to aid in the diagnosis of non-neoplastic and neoplastic bladder biopsies," Diagnostic Pathology, vol. 4, article 35, 2009.

[7] L. P. Kunju, C. T. Lee, J. Montie, and R. B. Shah, "Utility of cytokeratin 20 and Ki-67 as markers of urothelial dysplasia," Pathology International, vol. 55, no. 5, pp. 248-254, 2005. 
[8] J. K. McKenney, S. Desai, C. Cohen, and M. B. Amin, "Discriminatory immunohistochemical staining of urothelial carcinoma in situ and non-neoplastic urothelium: an analysis of cytokeratin 20, p53, and CD44 antigens," The American Journal of Surgical Pathology, vol. 25, no. 8, pp. 1074-1078, 2001.

[9] C. Mallofré, M. Castillo, V. Morente, and M. Solé, "Immunohistochemical expression of CK20, p53, and Ki-67 as objective markers of urothelial dysplasia," Modern Pathology, vol. 16, no. 3, pp. 187-191, 2003.

[10] S. H. Hong, D. E. Misek, H. Wang et al., "Identification of a specific vimentin isoform that induces an antibody response in pancreatic cancer," Biomark Insights, vol. 1, pp. 175-183, 2006.

[11] J. Ivaska, H.-M. Pallari, J. Nevo, and J. E. Eriksson, "Novel functions of vimentin in cell adhesion, migration, and signaling," Experimental Cell Research, vol. 313, no. 10, pp. 2050-2062, 2007.

[12] E. Korsching, J. Packeisen, C. Liedtke et al., "The origin of vimentin expression in invasive breast cancer: epithelialmesenchymal transition, myoepithelial histogenesis or histogenesis from progenitor cells with bilinear differentiation potential?" Journal of Pathology, vol. 206, no. 4, pp. 451-457, 2005.

[13] W. A. Raymond and A. S.-Y. Leong, "Co-expression of cytokeratin and vimentin intermediate filament proteins in benign and neoplastic breast epithelium," The Journal of Pathology, vol. 157, no. 4, pp. 299-306, 1989.

[14] C. Y. Ngan, H. Yamamoto, I. Seshimo et al., "Quantitative evaluation of vimentin expression in tumour stroma of colorectal cancer," British Journal of Cancer, vol. 96, no. 6, pp. 986-992, 2007.

[15] X. Ding, Y. Wang, X. Ma et al., "Expression of HMGA2 in bladder cancer and its association with epithelial-to-mesenchymal transition," Cell Proliferation, vol. 47, no. 2, pp. 146-151, 2014.

[16] A. Jemal, T. Murray, E. Ward et al., "Cancer statistics, 2005," CA-A Cancer Journal for Clinicians, vol. 55, no. 1, pp. 10-30, 2005.

[17] V. E. Reuter, "The urothelial tract: renal pelvis, ureter, urinary bladder and urethra," in Diagnostic Surgical Pathology, S. S. Sternberg, Ed., p. 1864, Lippincott Williams \& Wilkins, Philadelphia, Pa, USA, 3rd edition, 1999.

[18] S. Desai, S. D. Lim, R. E. Jimenez et al., "Relationship of cytokeratin 20 and CD44 protein expression with WHO/ISUP grade in pTa and pT1 papillary urothelial neoplasia," Modern Pathology, vol. 13, no. 12, pp. 1315-1323, 2000.

[19] N. H. Bassily, C. J. Vallorosi, G. Akdas, J. E. Montie, and M. A. Rubin, "Coordinate expression of cytokeratins 7 and 20 in prostate adenocarcinoma and bladder urothelial carcinoma," American Journal of Clinical Pathology, vol. 113, no. 3, pp. 383388, 2000.

[20] P. Paliwal, D. Arora, and A. K. Mishra, "Epithelial mesenchymal transition in urothelial carcinoma: twist in the tale," Indian Journal of Pathology and Microbiology, vol. 55, no. 4, pp. 443449, 2012.

[21] J. Zhao, D. Dong, L. Sun, G. Zhang, and L. Sun, "Prognostic significance of the epithelial-to-mesenchymal transition markers e-cadherin, vimentin and twist in bladder cancer," International Brazilian Journal of Urology, vol. 40, no. 2, 2014. 


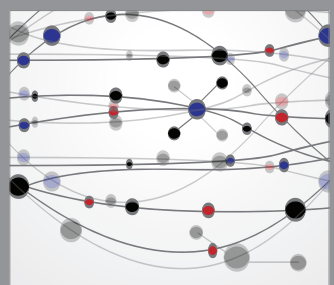

The Scientific World Journal
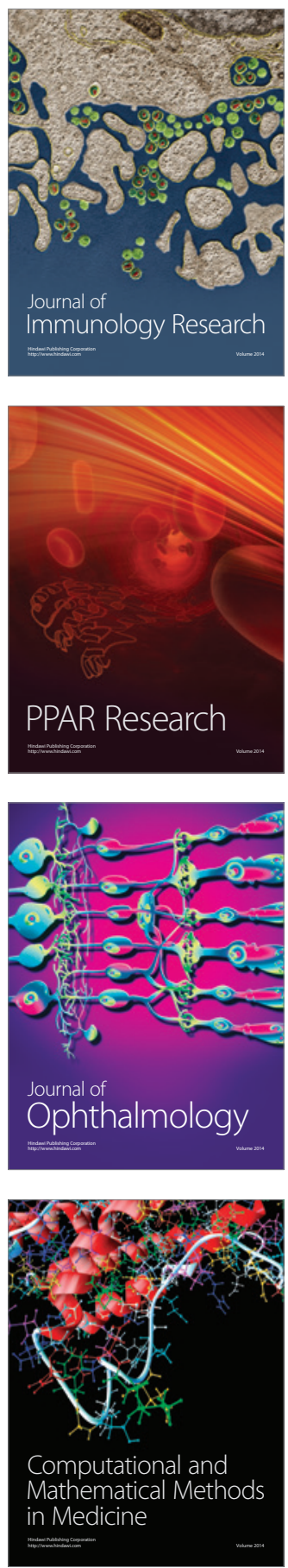

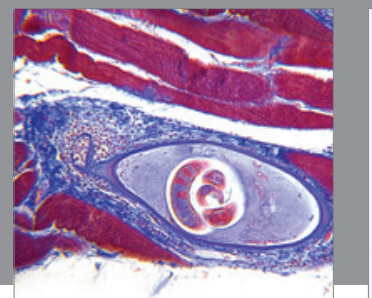

Gastroenterology

Research and Practice
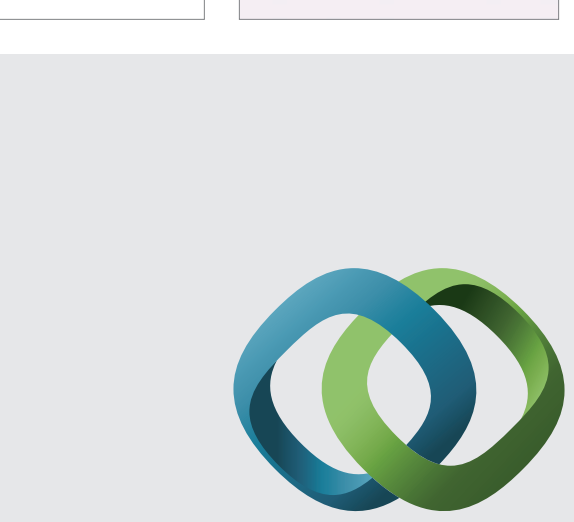

\section{Hindawi}

Submit your manuscripts at

http://www.hindawi.com
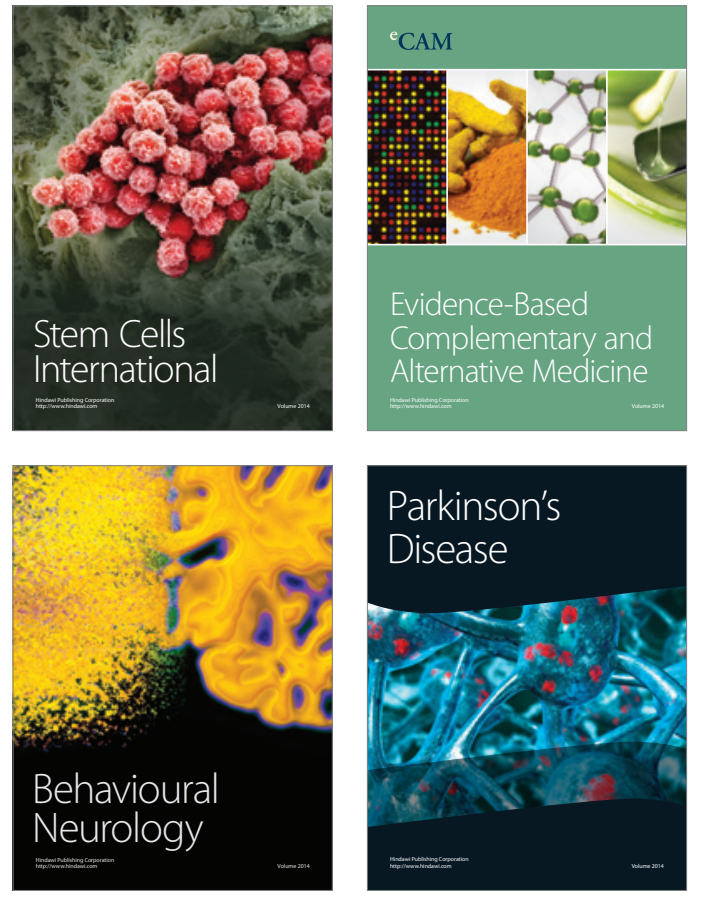
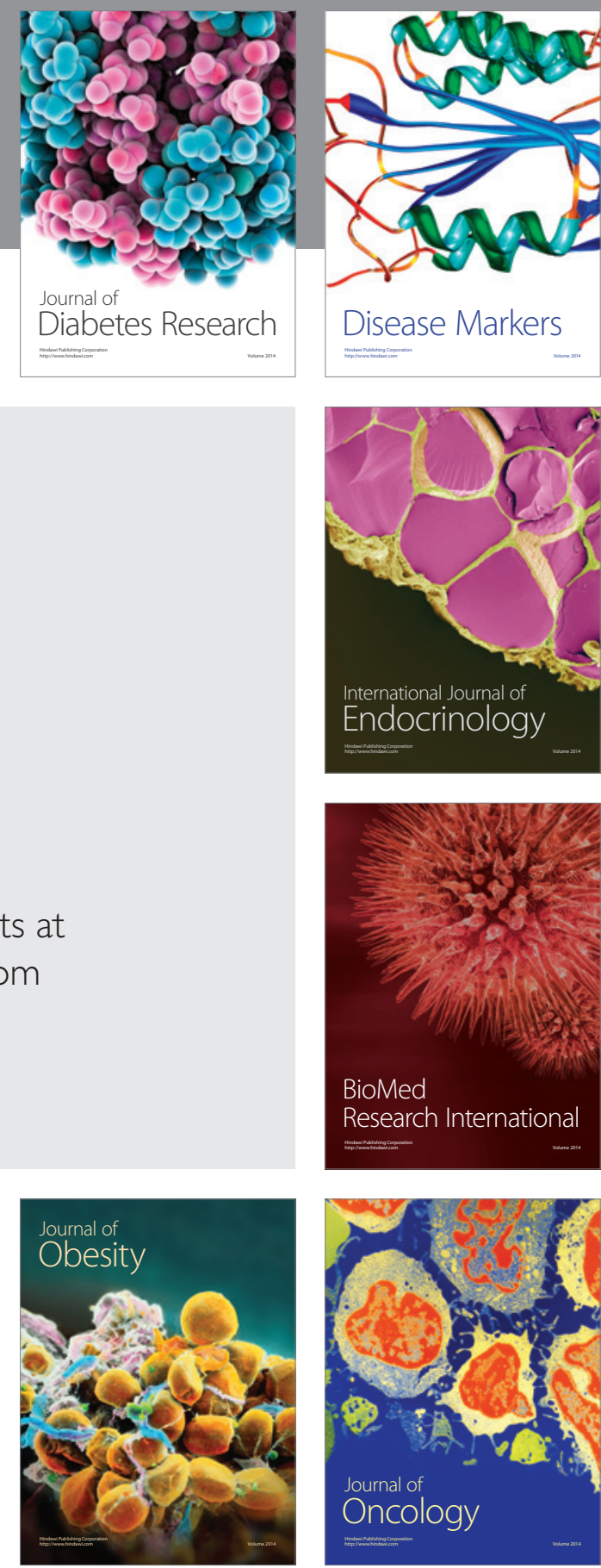

Disease Markers
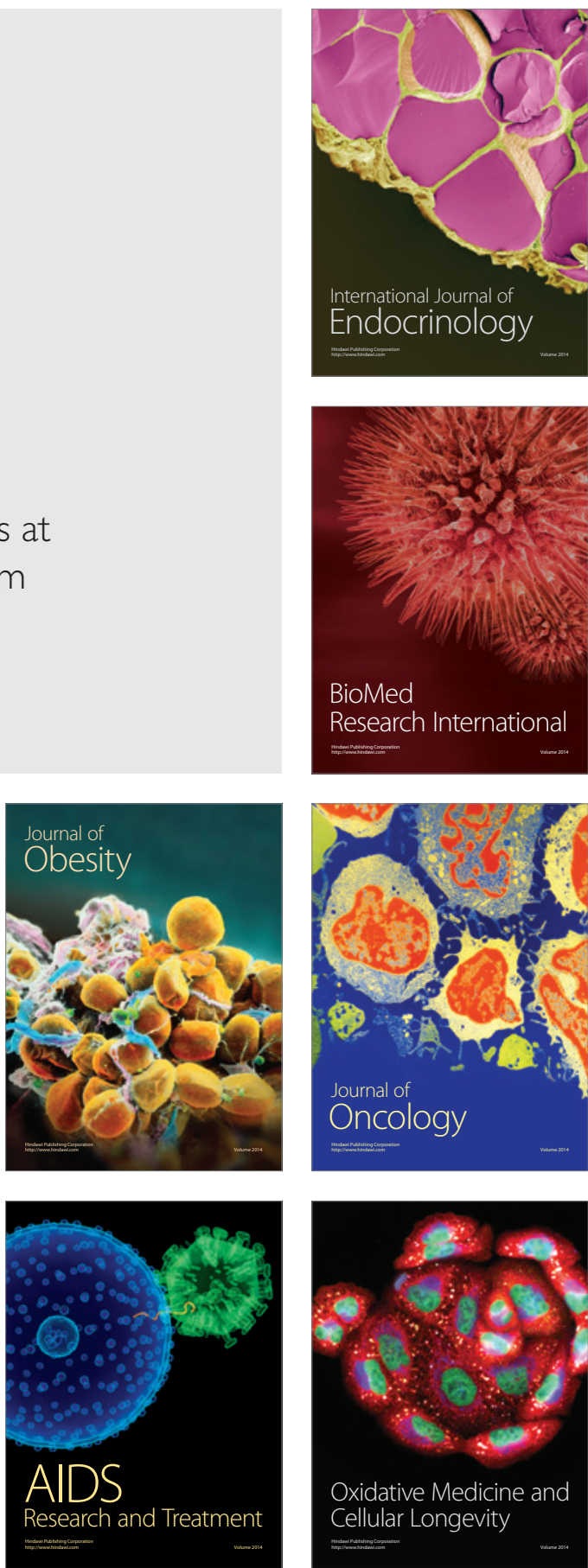